\title{
Evaluation of Clinical and Microbiological Characteristics of Foot Infections of Patients With Diabetes: Does Antibiotic Susceptibility Change?
}

\author{
Mehtap Evran ${ }^{\mathrm{a}, \mathrm{c}}$, Seher Cetinkaya Altuntas ${ }^{\mathrm{a}}$, Behice Kurtaran ${ }^{\mathrm{b}}$, \\ Murat Sert ${ }^{\mathrm{a}}$, Tamer Tetiker ${ }^{\mathrm{a}}$
}

\begin{abstract}
Background: The aim of the present study was to evaluate microorganisms isolated from foot ulcers of patients with diabetes, resistance profile and establish their relationship with amputation.

Methods: The study included 51 patients with diabetes who were admitted due to foot ulcer. The ulcers were evaluated according to the Wagner-Meggitt classification system. The specimens obtained by deep-tissue biopsy, aspiration biopsy and/or samples of pus from purulent lesions were sent for culture. Empiric antibiotic was started after collection of samples for culture. Antibiotic therapy was modified according to antibiotic susceptibility test results. The data were analyzed using the SPSS 20 software.
\end{abstract}

Results: Twelve females and 39 males were included in the study. The mean age of the patients was $63.1 \pm 10.9$ years, and duration of diabetes was $16.9 \pm 6.9$ years. Forty-six patients underwent amputation. The rate of amputation increased with the increasing Wagner grade $(\mathrm{P}=0.002)$. Neuropathy was found in $98 \%$ of the patients and in all patients that underwent amputation. About $97 \%$ of patients with osteomyelitis had undergone amputation, and these relationships were significant statistically $(\mathrm{P}=0.022)$. The most commonly isolated bacteria were as follows in order of frequency: E. faecalis $28(13.5 \%)$; S. aureus 23 (11\%); S. epidermidis 18 (8.6\%). Poly microbial infection was detected in 54 of a total of 138 episodes of our patients. The rate of ciprofloxacin resistance was $35 \%$ in $S$. aureus and $40-74 \%$ in Gram-negative bacteria. The rate of clindamycin resistance was $47 \%$ in $S$. aureus.

Conclusions: In our study, the most commonly isolated microorganisms exhibited a high rate of antibiotic resistance.

Manuscript submitted August 9, 2018, accepted August 28, 2018

aDivision of Endocrinology, Department of Internal Medicine, Cukurova University Medical Faculty, Adana, Turkey

bDepartment of Infectious Diseases and Clinical Bacteriology, Cukurova University Medical Faculty, Adana, Turkey

${ }^{\mathrm{c} C o r r e s p o n d i n g ~ A u t h o r: ~ M e h t a p ~ E v r a n, ~ D i v i s i o n ~ o f ~ E n d o c r i n o l o g y, ~ D e p a r t-~}$ ment of Internal Medicine, Cukurova University Medical Faculty, 01330, Sarıcam, Adana, Turkey. Email: mevran@cu.edu.tr

doi: https://doi.org/10.14740/jem525w
Keywords: Diabetes mellitus; Foot ulcer; Infection; Bacteria; Antibiotic susceptibility; Amputation

\section{Introduction}

Chronic hyperglycemia in patients with diabetes impairs cellular immunity and neutrophil functions, leading to insufficient chemotaxis and phagocytosis, and thus it paves the way for the development of fungal and bacterial infections [1,2]. Foot ulcers of patients with diabetes developing as a result of neuropathy also facilitate the entry of microorganisms into the body and their proliferation [3]. However, a poly microbial growth pattern involving Gram-positive and Gram-negative aerobic and anaerobic microorganisms occurs in severe foot infections of patients with diabetes $[4,5]$. These infections lead to the development of osteomyelitis and limb loss if the infection is not treated properly [6]. For this reason, causative microorganisms associated with foot infections of patients with diabetes must be recognized early, and appropriate therapy must be initiated immediately. The aim of the present study is to provide patients with proper antimicrobials considering antibiotic susceptibility profile of the identified microorganisms.

\section{Patients and Methods}

\section{Selection of the patients}

The study included 55 patients, who were admitted to our clinic due to foot ulcers of patients with diabetes. However, some essential information was not available in four patients, and thus the study was conducted with the findings of 51 patients.

\section{Clinical evaluations and descriptions}

Age, gender, and the type and duration of diabetes of the patients were recorded. The presence of chronic complications such as neuropathy, nephropathy and retinopathy were also noted. In addition, plasma levels of glucose and glycosylated 
hemoglobin $(\mathrm{HbA} 1 \mathrm{c})$ were measured upon admission, and the results were recorded.

Neurological examination of the superficial and deep sensations (vibration sense and position sense) was performed using a 128-Hertz diapason, and deep tendon reflexes were evaluated. Some patients also underwent electromyography (EMG) for the evaluation of neuropathy. Micro albumin levels in 24-h urine were measured to evaluate patients for nephropathy, and the results were graded as follows: $<30 \mathrm{mg}$, normal; 30 - $300 \mathrm{mg}$, micro-albuminuria; > $300 \mathrm{mg}$, macro-albuminuria [7]. Vascular assessment was performed using Doppler ultrasonography (US), magnetic resonance angiography (MRA) or conventional angiography. In addition to clinical assessment, direct graphs and/or magnetic resonance imaging (MRI) were used as imaging methods to evaluate patients for the presence of osteomyelitis. The presence of bone erosions or local demineralization in association with soft tissue infection in direct graphs was considered to the favor of osteomyelitis.

Intensive insulin therapy was initiated in all patients for the regulation of plasma glucose level. Foot ulcers of patients with diabetes were evaluated according to the Wagner-Meggitt classification system [8]. The amputations performed at the level of the distal phalanx and phalanx and/or metatarsus were considered minor amputations, and those performed at the level of the heel, ankle, below-knee and above-knee were considered major amputations.

In the presence of more than one foot infection in the same patient, the infectious episodes occurring in the patients were separately included in the study. It was stipulated that a new situation was to be considered a new episode if the previous episode has recovered or concluded with amputation.

The specimens obtained by deep-tissue biopsy, aspiration biopsy and/or samples of pus from purulent lesions were sent for culture. At least two sets of blood cultures were taken when the patients exhibited symptoms of a systemic infection. Empirical antibiotic treatment was initiated according to the clinical condition of the patient after obtaining samples for culture. Initial empiric antibiotic therapy was modified according to antibiotic susceptibility test results.

\section{Microbiological method}

The microorganisms isolated from the wound cultures and their antibiotic susceptibility profiles were evaluated retrospectively in the study. The specimens of deep tissue and swab samples taken following debridement, samples of abscess and drainage, aspirates, tissue biopsies, and the samples of superficial wound swabbing were included in the study. The samples were collected after cleaning the surface with sterile serum physiologic solution in open lesions and after cleaning with $70 \%$ alcohol in closed lesions [9-11]. The samples were transported to the laboratory in the Amies transport medium (bioMerieux), the thioglycollate broth medium (bioMerieux), or in the sterile sample container. For cultivation, the specimens were inoculated on 5\% sheep blood agar (COS; bioMerieux), chocolate agar (PVX; bioMerieux), MacConkey agar (MCK; bioMerieux), and Sabouraud dextrose agar (SDA; bioMerieux). The plaques were incubated in an incubator at $37^{\circ} \mathrm{C}$ for $18-24$ $\mathrm{h}$ and were then evaluated for bacterial growth. The plaques showing no bacterial growth were kept in the incubator for up to $96 \mathrm{~h}$, and they were evaluated for bacterial growth at the end of this time period. The thioglycollate broth (bioMerieux) media were also incubated in the incubator at $37^{\circ} \mathrm{C}$ for $96 \mathrm{~h}$, and they were evaluated daily for the growth of bacteria $[9,10]$.

The conventional methods (morphology of bacterial colony, Gram staining, catalase test, oxidase test, plasma coagulase test for staphylococci), and the Vitek 2 system cards for the identification of Gram-positive and Gram-negative bacteria (GN ID and GP ID, bioMerieux) were used for the identification of microorganisms reproducing in clinical samples.

Extended spectrum beta lactamase (ESBL) production by Escherichia coli (E. coli) and Klebsiella pneumoniae (K. pneumonia) species was detected by the Vitek 2 AES (The Advanced Expert System ${ }^{\mathrm{TM}}$, bioMerieux) system.

Antibiotic susceptibility tests for Gram-positive and Gram-negative bacteria were performed using the Vitek 2 automated system (bioMerieux); the results of minimal inhibitory concentration (MIC) were graded as susceptible, intermediate or resistant according to the guidelines of Clinical and Laboratory Standards Institute (CLSI) [12].

\section{Statistical analysis}

The data were analyzed using SPSS 20 software. The categorical variables were expressed as numbers and percentage, and numeric variables were expressed as mean and standard deviation (median and minimum - maximum where required). The Chi-square test was used to compare categorical variables between the groups. The Kolmogorov-Smirnov test was used to test the hypothesis that numeric variables were normally distributed. In comparison of the numeric variables between the groups, the independent samples $t$-test was used when the hypothesis was accepted, and the Mann-Whitney U test was used when the hypothesis was rejected. The level of statistical significance was set at $\mathrm{P}<0.05$ in all statistical analyses.

\section{Results}

The study included 51 patients with type 2 diabetes mellitus (T2DM), who were admitted to our clinic due to foot ulcer. Of these patients, 12 were female $(23.5 \%)$ and 39 were male $(77.5 \%)$. The mean age was $63.1 \pm 10.9$ years, and the mean duration of diabetes was $16.9 \pm 6.9$ years (min - max: 4 - 35 years). Demographic characteristics of the patients and presence of complications are demonstrated in Table 1.

Forty-six patients underwent amputation (22 minor, 24 major). When the amputations were evaluated in terms of gender, the number of amputations did not differ between men and women $(\mathrm{P}=0.32)$, while the type of amputation differed significantly. The frequency of minor amputations was found to be higher in female patients $(75 \%)$, while the frequency of major amputations was higher in males $(65 \%)(\mathrm{P}=0.021)$. The mean age was $63.8 \pm 10.3$ years in patients, who underwent amputation, and $59.4 \pm 15.2$ years in patients, who did not 
Table 1. Demographic Characteristics of the Patients and Presence of Chronic Complications

\begin{tabular}{lll}
\hline & N $(\%)$ & Mean \pm SD \\
\hline $\begin{array}{l}\text { Age (year) } \\
\text { Duration of diabetes (year) }\end{array}$ & $63.1 \pm 10.9$ \\
Gender & $16.9 \pm 6.9$ \\
$\quad$ Female & $12(23.5 \%)$ & \\
$\quad$ Male & $39(77.5 \%)$ & \\
Neuropathy & $50(98 \%)$ \\
Nephropathy & $44(86 \%)$ \\
Retinopathy & $48(94 \%)$ \\
Peripheral arterial disease & $40(78.4 \%)$ \\
Wagner stage* & \\
$\quad$ Wagner 2 & $5(9.6 \%)$ \\
$\quad$ Wagner 3 & $33(63.5 \%)$ \\
$\quad$ Wagner 4 & $14(26.9 \%)$ \\
\hline Osteomyelitis & $47(90.4 \%)$ \\
Amputation & $46(90.1 \%)$ \\
\hline
\end{tabular}

*No patients in Wagner stages 0 and 1.

undergo amputation; this difference was not statistically significant $(\mathrm{P}=0.430)$. The duration of diabetes was found to be higher in patients who underwent amputation, when compared to those who did not undergo amputation; however this difference was not found to be statistically significant.

A significant relationship existed between the Wagner grade of the foot ulcers and amputation. The rate of amputation increased with increasing Wagner grade $(\mathrm{P}=0.002$; the rates of amputation in Wagner 2, Wagner 3 and Wagner 4 were $40 \%$, $94.1 \%$, and $100 \%$, respectively).

When the patients were evaluated in terms of chronic complications of diabetes, neuropathy was detected in $98 \%$ of the patients, and in all patients that underwent amputation. Amputation was performed in $97 \%$ of patients with osteomyelitis, $93.8 \%$ of patients with retinopathy, and these relationships were statistically significant $(\mathrm{P}=0.022$ and $\mathrm{P}=0.023$, respectively). A significant relationship was not found between the presence of peripheral arterial disease and amputation $(\mathrm{P}=0.580)$.

The results of cultures obtained from the patients revealed bacterial growth in a total of 207 samples. Forty-three different bacteria were isolated from these cultures. The most commonly isolated bacteria were as follows in the order of frequency: Enterococcus faecalis (E. faecalis), 28 (13.5\%); Staphylococcus aureus (S. aureus), 23 (11\%); Staphylococcus epidermidis (S. epidermidis), 18 (8.6\%); Pseudomonas aeruginosa (P. aeruginosa), 16 (7.7\%); Morganella morganii (M. morganii), 15 (7.2\%); and E. coli, 13 (6.2\%). There was polymicrobial growth in 54 cultures; of these, eight showed growth of three microorganisms and one showed growth of four microorganisms. The reproducing microorganisms in the collected samples are indicated in Table 2. Mono microbial and poly microbial growth groups, their Gram-staining characteristics, and the rates of amputation in each group are demonstrated in Table 3 , and the
Table 2. Types of Microorganisms Identified in the Wound Cultures of the Patients

\begin{tabular}{|c|c|}
\hline Bacteria & Total (N) \\
\hline Gram-positive microorganisms & 96 \\
\hline Enterococcus faecalis & 28 \\
\hline Staphylococcus aureus & 23 \\
\hline Staphylococcus epidermidis & 18 \\
\hline Other CNS & 9 \\
\hline Streptococcus agalactiae & 8 \\
\hline Streptococcus dysgalactiae & 4 \\
\hline Streptococcus viridans & 3 \\
\hline Enterococcus avium & 2 \\
\hline Streptococcus pyogenes & 1 \\
\hline Gram-negative microorganisms & 101 \\
\hline Pseudomonas aeruginosa & 16 \\
\hline Morganella morganii & 15 \\
\hline Escherichia coli & 13 \\
\hline Acinetobacter baumannii & 11 \\
\hline Proteus mirabilis & 11 \\
\hline Serratia marcescens & 6 \\
\hline Klebsiella pneumoniae & 5 \\
\hline Citrobacter koseri & 4 \\
\hline Providencia rettgeri & 3 \\
\hline Klebsiella oxytoca & 2 \\
\hline Citrobacter braakii & 2 \\
\hline Citrobacter freundii & 2 \\
\hline Enterobacter cloacae & 2 \\
\hline Stenotrophomonas maltophilia & 3 \\
\hline Proteus penneri & 1 \\
\hline Proteus vulgaris & 1 \\
\hline Providencia alcalifaciens & 1 \\
\hline Providencia stuartii & 1 \\
\hline Serratia liquefaciens & 1 \\
\hline Sphingomonas paucimobilis & 1 \\
\hline \multicolumn{2}{|l|}{ Fungi } \\
\hline Candida species & 1 \\
\hline Total & 207 \\
\hline
\end{tabular}

susceptibility profile of these bacteria is presented in Table 4.

\section{Discussion}

Infection appears to be the result, and not the cause of foot ulcers of patients with diabetes. Foot ulcers are often accompanied by infectious agents, which lead to a wide-spectrum of infections from a simple infection as paronychia and cellulitis 
Table 3. The Rates of Amputation in Regard to Mono Microbial and Poly Microbial Reproductions, and Gram-Staining Properties of the Pathogens

\begin{tabular}{llll}
\hline Characteristic of reproduction & Number (N) & Amputation N (\%) & Osteomyelitis N (\%) \\
\hline Mono microbial & 85 & $74(87 \%)$ & $54(64 \%)$ \\
Gram-positive & 43 & & \\
Gram-negative & 42 & $52(98 \%)$ & $40(75 \%)$ \\
Poly microbial & 54 & & \\
All are Gram-positive & 12 & & \\
All are Gram-negative & 7 & & \\
Mixed & 35 &
\end{tabular}

to a more severe condition as osteomyelitis [13]. The presence of osteomyelitis is associated with poor prognosis, and it also increases amputation risk considerably by making foot ulcer a difficult condition to treat [14]. Therefore, it is extremely important to identify infectious agents early in foot ulcers of patients with diabetes and administer the treatment accordingly. The use of proper antibiotics prevents the progression of ulcer, and thus, avoids loss of extremity and patient's life [15].

In patients with diabetes, the entry of bacteria occurs through acute traumatic wounds, burns, fissures between the fingers, and chronic pressure wounds in which the integrity of normal skin barrier has been interrupted. The infection occurring with this mechanism may be either localized or wide and deep [16]. Acute foot infections in patients with diabetes are mono microbial similar to those observed in patients without diabetes, and responsible agent is $S$. aureus or especially group B streptococci $[13,17]$. Chronic infections, however, can be poly microbial and the dominant microorganisms in such infections are expected to be $S$. aureus followed by group B streptococci. The patients may require aggressive surgical debridement due to potential of these microorganisms to cause a necrotizing infection [16]. Gram-positive microorganisms such as enterococci and Gram-negative bacilli such as E. coli, Klebsiella and Proteus may reproduce in patients, who have previously received antibiotics (especially cephalosporins). Many antibiotic-resistant species of Pseudomonas can be detected especially in sweaty feet or inter digital macerations $[18,19]$. The detection of coagulase-negative Staphylococci (CNS) in super-

Table 4. Antibiotic Sensitivity Patterns of Microorganisms Identified in Culture Media

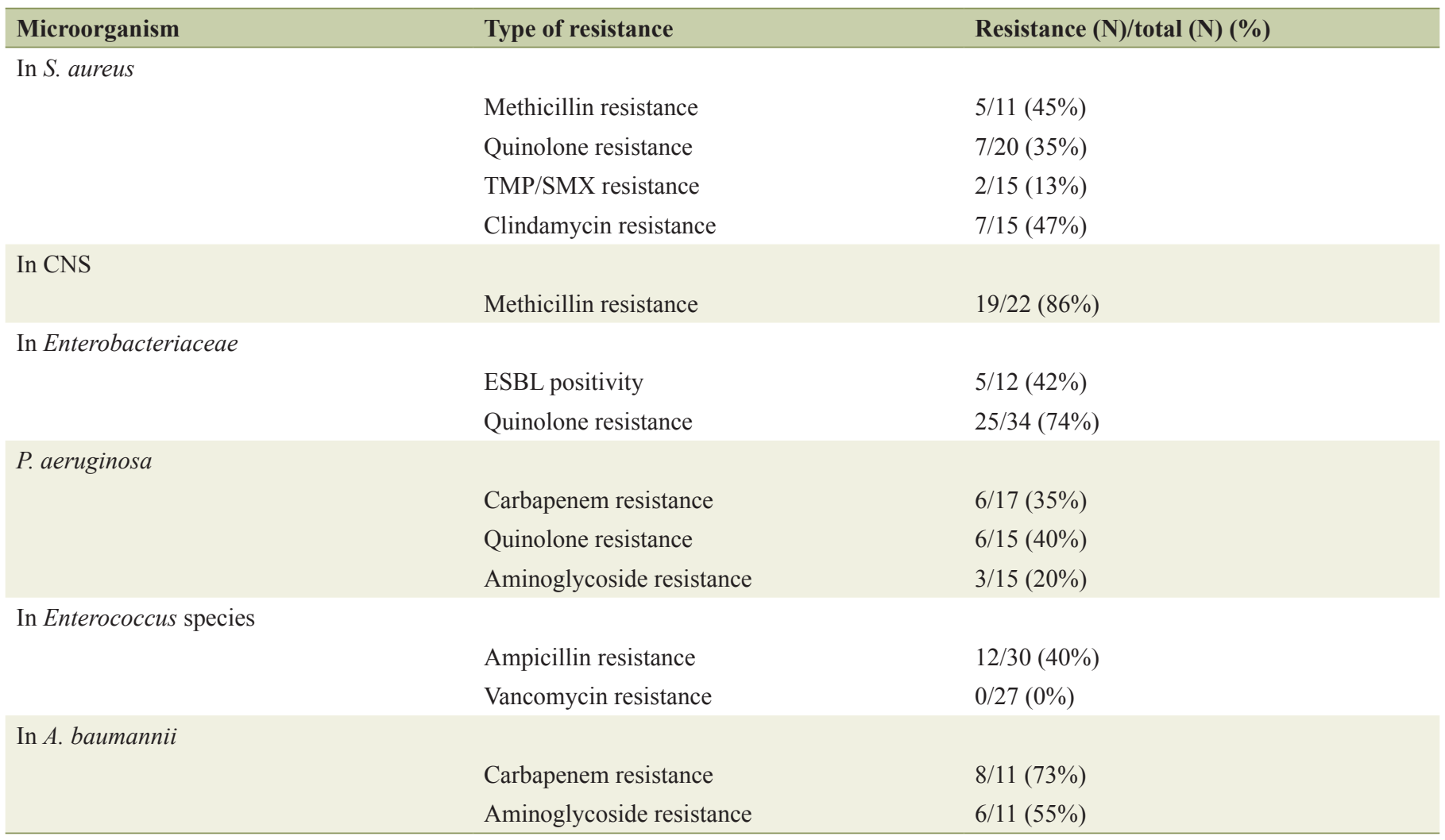


ficial wound infections generally indicates the presence of contamination, while detection in cultures of deep tissues and bone cultures points to a condition that needs to be taken seriously and treated immediately. Candida infections especially lead to tinea pedis, and they can be detected in some cases of foot ulcers of patients with diabetes. Bacteroides fragilis ( $B$. fragilis) is an anaerobic microorganism that can be mostly detected in necrotic wounds or ischemic wounds of the foot $[20,21]$.

The most commonly reproducing bacteria in patients included in our study were as follows in the order of frequency: E. faecalis, $S$. aureus, $S$. epidermidis, $P$. aeruginosa, and $M$. morganii. In a study that was conducted at our clinic approximately 15 years ago by the follow-up of 100 patients with foot ulcers of patients with diabetes, $S$. aureus was found to be the most commonly reproducing bacteria [22]. Of a total of 138 infectious episodes in patients in the present study, poly microbial reproduction was determined in 54 (39\%) culture results; of these, eight showed growth of three microorganisms, one showed growth of four microorganisms, and two microorganisms were detected in the remaining. When long years are taken into consideration, we may state that microorganisms reproducing in our patients are generally similar; however, the frequency has changed, and E. faecalis, which ranked third in previous years, has taken the first place in the present study. In the case of microorganisms such as Staphylococcus and Enterococcus that are often associated with contamination, isolation of these agents from repeated cultures and consistency with clinical picture helped differentiation. The response to pathogen-specific treatment has also supported that they were the responsible pathogens. Most of our patients were referred from external centers, and this fact may be a reason for the reproduction of pathogens (such as the species of Enterococcus), which are not covered by therapeutic spectrum of the therapies administered in the referring centers [13].

The relationship between reproducing microorganisms and amputation could not be evaluated, since most of the patients in our study population underwent amputation, poly microbial reproductions were present, and the patients exhibited with more than one infectious episode.

In patients followed due to foot ulcers of patients with diabetes, it is an appropriate approach to administer empiric antibiotic therapy until the results of culture becomes available. However, empiric antibiotic regimen selected should cover the most commonly isolated microorganisms [16]. Combined antibiotic therapies must be selected considering poly microbial disease [13]. High rates of resistance in the pathogens may be related to high number of advanced-stage patients in the present study, and thus possibly to the history of intensive antibiotic use for this purpose. In addition, frequent hospitalization is an important risk factor for colonization of and infection with resistant microorganisms. Combination of ciprofloxacin and clindamycin is frequently used at our hospital in oral empiric therapy for foot infections of patients with diabetes. In the present study, the rate of resistance against ciprofloxacin was $35 \%$ in $S$. aureus and between $40 \%$ and $74 \%$ in Gram-negative bacteria. Clindamycin resistance was also found to be high in $S$. aureus, which was at a rate of $47 \%$. Although the number of our patients was not sufficient to draw a conclusion, high rates of resistance to these drugs attract attention and have to be taken into consideration $[13,23]$.

Osteomyelitis develops in about two thirds in foot ulcers of patients with diabetes, and the presence of osteomyelitis increases the risk of amputation considerably by making foot ulcer a difficult condition to treat [15]. Nevertheless, there are studies indicating that osteomyelitis in foot ulcers of patients with diabetes might recover with antibiotic therapy without requiring surgical intervention. Therefore, in the presence of osteomyelitis, antibiotic regimen must be selected carefully [2426]. The frequency of osteomyelitis was $90 \%$ in our patients, and this result was found to be high compared to our previous results. The rate of amputation was also found to increase with the increasing Wagner stage in our patients. It would be appropriate to mention that we found a high rate of amputation, since our study population was comprised of patients with advanced stages of foot ulcer who were referred to our clinic from other centers within the last few months.

A significant relationship was not detected between the presence of peripheral arterial disease and amputation in our patients, while neuropathy, retinopathy and osteomyelitis were found to be associated with amputation.

The most important limitations of our study are insufficient number of patients, and small number of patients who did not undergo amputation. Poly microbial growth and more than one infectious episode occurring in the study participants complicate evaluation of the relationship between antibiotic susceptibility and amputation.

In conclusion, E. faecalis and $S$. aureus are the most frequently isolated microorganisms in the present study. The results of antibiotic susceptibility tests guide selection of appropriate empiric antibiotic therapy; however, these data are insufficient to change current approaches due to small number of patients. The ideal approach in preventing osteomyelitis and/or decreasing amputations through appropriate treatment of osteomyelitis would be to wait for culture results and administer agent-specific antibiotics.

\section{Acknowledgments}

We thank to both the Department of Microbiology and the Central Laboratory of our hospital.

\section{Funding}

None.

\section{Conflict of Interest}

None.

\section{Author Contributions}

Research conception and design: Evran M. Data acquisition: Cetinkaya Altuntas S, Evran M and Kurtaran B. Data analysis 
and interpretation: Evran M and Kurtaran B. Statistical analysis: Evran M. Drafting of the manuscript: Evran M, Cetinkaya Altuntas S, Kurtaran B and Tetiker T. Critical revision of the manuscript: Tetiker T, Sert Murat. Approval of final manuscript: all authors.

\section{References}

1. Kosinski ME, Lipsky BA. Current medical management of diabetic foot infection. Expert Rev Anti Infect Ther. 2010;8(11):1293-1305.

2. Delamaire M, Maugendre D, Moreno M, Le Goff MC, Allannic H, Genetet B. Impaired leucocyte functions in diabetic patients. Diabet Med. 1997;14(1):29-34.

3. Lipsky BA, Berendt AR. Principles and practice of antibiotic therapy of diabetic foot infections. Diabetes Metab Res Rev. 2000;16(Suppl 1):S42-46.

4. Lipsky BA. A report from the international consensus on diagnosing and treating the infected diabetic foot. Diabetes Metab Res Rev. 2004;20(Suppl 1):S68-77.

5. Pellizzer G, Strazzabosco M, Presi S, Furlan F, Lora L, Benedetti P, Bonato M, et al. Deep tissue biopsy vs. superficial swab culture monitoring in the microbiological assessment of limb-threatening diabetic foot infection. Diabet Med. 2001;18(10):822-827.

6. Caballero E, Frykberg RG. Diabetic foot infections. J Foot Ankle Surg. 1998;37(3):248-255.

7. Zelmanovitz T, Gerchman F, Balthazar AP, Thomazelli FC, Matos JD, Canani LH. Diabetic nephropathy. Diabetol Metab Syndr. 2009;1(1):10.

8. Wagner FW, Jr. The dysvascular foot: a system for diagnosis and treatment. Foot Ankle. 1981;2(2):64-122.

9. Baron EJ, Thomson RB, Miller JM. Specimen collection, transport and processing: Bacteriology. In: Versalovic J, ed. Manuel of Clinical Microbiology, American Society for Microbiology 10th edn., Washington D.C.; 2011, p. 228-271.

10. Murray PR. The Clinician and the Microbiology Laboratory. In: Bennett JE, Dolin R, Blaser MJ, eds. Mandell, Douglas, and Bennett's Principles and Practice of Infectious Disease, 8th ed. Philadelphia, Elseiver Saunders. 2015; p. 191-223.

11. Wilson ML, Winn W. Laboratory diagnosis of bone, joint, soft-tissue, and skin infections. Clin Infect Dis. 2008;46(3):453-457.

12. Clinical and Laboratory Standards Institute (CLSI). Performance standard for antimicrobial susceptibility testing. Twenty second informational supplement. Document 2012; 31(1): M100-S22 CLSI, Wayne, PA, USA.

13. Zubair M, Malik A, Ahmad J. Microbiology of diabetic foot ulcer with special reference to ESBL infections.
American journal of Clinical and Experimental Medicine. 2015;3(1):6-23.

14. Reiber GE, Lipsky BA, Gibbons GW. The burden of diabetic foot ulcers. Am J Surg. 1998;176(2A Suppl):5S$10 \mathrm{~S}$.

15. Grayson ML, Gibbons GW, Balogh K, Levin E, Karchmer AW. Probing to bone in infected pedal ulcers. A clinical sign of underlying osteomyelitis in diabetic patients. JAMA. 1995;273(9):721-723.

16. Kosinski MA, Lipsky BA. Current medical management of diabetic foot infections. Expert Rev Anti Infect Ther. 2010;8(11):1293-1305.

17. Ramakant P, Verma AK, Misra R, Prasad KN, Chand G, Mishra A, Agarwal G, et al. Changing microbiological profile of pathogenic bacteria in diabetic foot infections: time for a rethink on which empirical therapy to choose? Diabetologia. 2011;54(1):58-64.

18. Lipsky BA, Pecoraro RE, Larson SA, Hanley ME, Ahroni JH. Outpatient management of uncomplicated lower-extremity infections in diabetic patients. Arch Intern Med. 1990;150(4):790-797.

19. Sapico FL, Witte JL, Canawati HN, Montgomerie JZ, Bessman AN. The infected foot of the diabetic patient: quantitative microbiology and analysis of clinical features. Rev Infect Dis. 1984;6(Suppl 1):S171-176.

20. Dang CN, Prasad YD, Boulton AJ, Jude EB. Methicillinresistant Staphylococcus aureus in the diabetic foot clinic: a worsening problem. Diabet Med. 2003;20(2):159-161.

21. Eady EA, Cove JH. Staphylococcal resistance revisited: community-acquired methicillin resistant Staphylococcus aureus - an emerging problem for the management of skin and soft tissue infections. Curr Opin Infect Dis. 2003;16(2):103-124.

22. Sert M, Tetiker T, Koçak M. Cukurova yoresinde diyabetik ayak yarasi olan hastalarin klinik seyri. Endokrinolojide Yonelişler. 2004;8(1).

23. Bowler PG. The 10(5) bacterial growth guideline: reassessing its clinical relevance in wound healing. Ostomy Wound Manage. 2003;49(1):44-53.

24. Raymakers JT, Houben AJ, van der Heyden JJ, Tordoir JH, Kitslaar PJ, Schaper NC. The effect of diabetes and severe ischaemia on the penetration of ceftazidime into tissues of the limb. Diabet Med. 2001;18(3):229-234.

25. Legat FJ, Maier A, Dittrich P, Zenahlik P, Kern T, Nuhsbaumer S, Frossard M, et al. Penetration of fosfomycin into inflammatory lesions in patients with cellulitis or diabetic foot syndrome. Antimicrob Agents Chemother. 2003;47(1):371-374.

26. Oberdorfer K, Swoboda S, Hamann A, Baertsch U, Kusterer K, Born B, Hoppe-Tichy T, et al. Tissue and serum levofloxacin concentrations in diabetic foot infection patients. J Antimicrob Chemother. 2004;54(4):836-839. 